\title{
Influence of copper layer on epoxy acrylate based solder mask surface chemistry and the effect on epoxy adhesion
}

\author{
Caroline Hofmeister ${ }^{1,2^{*}}$, Sebastian Maaß ${ }^{1}$, Thorsten Fladung ${ }^{2}$, Karsten Thiel ${ }^{2}$ and Bernd Mayer ${ }^{2}$
}

*Correspondence:

caroline.hofmeister@

de.bosch.com

1 Robert Bosch $\mathrm{GmbH}$

Postfach 3002 40,

70442 Stuttgart, Germany

Full list of author information

is available at the end of the

article

\begin{abstract}
The influence of the presence of copper on the network formation and the surface chemistry of an epoxy acrylate based solder mask system were investigated, with regards to the application of solder masks on copper layer. The presence of copper within the bulk volume of the solder mask was characterized by utilizing scanning electron microscopy/focused ion beam and energy dispersive X-ray analysis. The copper originates from the copper layer underneath and decreases towards the surface. Differences in the network structure were analyzed by applying infrared spectroscopy in attenuated total reflection mode, which showed no changes in the network structure due to a copper layer underneath. The surface chemistry of two solder masks with different curing agents, amine and anhydride, was investigated for differences caused by changes in the curing behavior depending on copper complex formation. Surface chemistry was analyzed by applying X-ray photoelectron spectroscopy and time of flight secondary ion mass spectrometry. Measurable differences in the surface chemistry with regards to copper content are generated due to the copper layer underneath. However, through the optical contact angle method and pull-off test, no differences concerning wetting or adhesion behavior between the solder mask and an epoxy adhesive were observed.
\end{abstract}

Keywords: Solder mask, Adhesion, XPS, ToF-SIMS, Printed circuit board

\section{Background}

Epoxy acrylate based solder masks are used in printed circuit board (PCB) technologies, providing defined solder pad geometries and mechanically protected circuitry. During production of an electronic device, adhesive bonding of different adhesion partners is applied, such as thermally conductive materials, sealing adhesives and encapsulations to the PCB surface. In this case, the solder mask provides the surface on which adhesion is supposed to occur. Since the solder mask is applied on the whole PCB surface, consisting of epoxy base material as well as of copper, intimate contact to copper surfaces is given. With regard to the adhesion on solder masks in the PCB composite, this study focusses on photoimageable solder masks based on epoxy acrylate dual cure systems [1].

It is known that the acrylic component reacts UV initiated in the presence of photoinitiators in a radical polymerization reaction, while the epoxy component cures heat activated in a polyaddition reaction [2]. The curing components might be aliphatic or

(c) 2015 Hofmeister et al. This article is distributed under the terms of the Creative Commons Attribution 4.0 International License (http://creativecommons.org/licenses/by/4.0/), which permits unrestricted use, distribution, and reproduction in any medium, provided you give appropriate credit to the original author(s) and the source, provide a link to the Creative Commons license, and indicate if changes were made. 
aromatic amines, anhydrides (requiring imidazoles as accelerators [3]) or novolak compounds [4]. The formation of copper complex species in an epoxy resin system utilizing amine or anhydride hardeners is well known and has been documented in literature [59]. Hamerton et al. [7] described an accelerated reaction of epoxy with copper-amine complexes leading to higher conversions of functional groups. Roche et al. [8] described the expansion of an epoxy-diamine/metal interphase (approx. 200-600 $\mu \mathrm{m}$ ) depending on parameters like layer thickness, temperature, time, hardener type and mix ratio. Considering the curing reaction of epoxy and anhydride, the contrary effect can occur. Anhydrides exhibit slow curing kinetics below temperatures of $200{ }^{\circ} \mathrm{C}$. For moderate curing conditions, accelerators such as imidazoles are added [3]. These imidazoles are known to form copper imidazole complexes [10-12], thus, the imidazole reacts with the copper in a coordination reaction and not as an accelerator in the ring opening reaction with the anhydride. Accelerated or decelerated network formation due to the presence of amine or anhydride hardeners via complexation of copper leads to different curing states compared to systems where copper is not present.

Due to the fact that the bulk volume affects the surface chemistry, which functions as contact zone where adhesion takes place, variations in the cross-linking density could generate (for example, in polar surface groups) if respective end groups are available due to an incomplete reaction. Additionally, the cross-linking density influences the migration behavior of low molecular weight compounds. Considering the different adhesion mechanisms of polymers [13], the evaluation of the surface chemistry as well as the influence of the presence of copper underneath the solder mask on epoxy adhesion, were the focuses of this study.

\section{Methods}

Materials

In this study, two different types of solder masks (A and B) applied on base material (SM-BM) or directly on bare copper (SM-Cu) were used. The configurations SM-BM and $\mathrm{SM}-\mathrm{Cu}$ are visualized in Fig. 1. Before the application of the solder mask on the substrates (base material or bare copper) takes place, these substrates are chemically or mechanically roughened, via micro-etching or pumice scrubbing [14]. The substrate treatment of base material, copper base layer and each solder mask type, was carried out under the same terms. The base material was conventional FR-4 laminate, consisting of glass fiber mats embedded with epoxy resin.

The basic chemistry of the solder masks was an epoxy acrylate dual cure system (bisphenol-A based epoxy and methyl methacrylate) with typical photoimageable solder

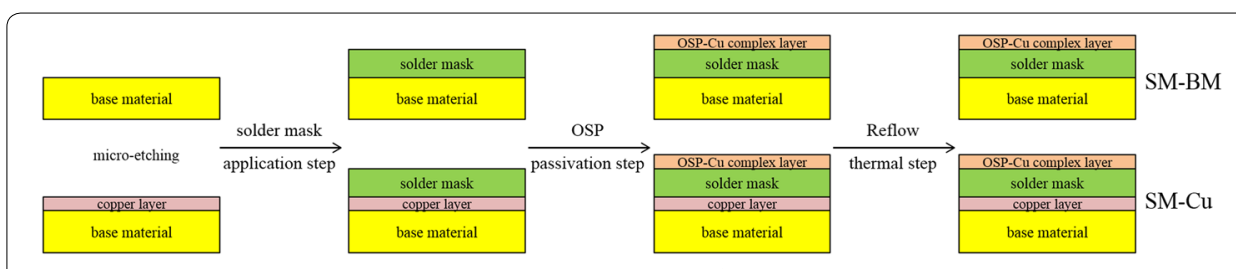

Fig. 1 Configuration and processing: structure of the two configurations: solder mask on base material (SM$\mathrm{BM})$ and solder mask on copper base layer (SM-Cu) 
mask formulations, including photoinitiators, thermal curing agents, silica-filler, pigment and several organic solvents. Solder mask type A was cured with an anhydride and solder mask type B was cured with an amine. Additionally, both solder mask formulations contained additives, like PDMS-based anti-foaming and wetting agents, which are introduced to impact specific properties of the solder masks. Anti-foaming agents contribute to the solder mask performance in a curtain coating application process [1].

To condition the solder mask surface in a typical PCB manufacturing and assembly process (see Fig. 1), solder mask surfaces were exposed to organic solderability preservative (OSP) bath chemistry during the passivation of bare copper areas on the $\mathrm{PCB}$ for corrosion inhibition during the PCB manufacturing process. To include the influence of this special wet bath chemistry in our research, the solder mask surfaces were treated with a commercially available OSP, based on benzimidazole chemistry $[15,16]$. Additionally, for PCB-conditioning, each sample was exposed to a two stage thermal pretreatment in a so-called "reflow oven" (Hotflow 2/24, ERSA GmbH, Germany). The temperature profile of the oven, shown in Fig. 2, exhibited a maximum temperature of $245^{\circ} \mathrm{C}$ on the PCB surface in a $1000 \mathrm{ppm}$ oxygen atmosphere. Line speed was $800 \mathrm{~mm} /$ min. This thermal treatment represents the soldering of electronic elements during $\mathrm{PCB}$ assembly. At the end of the above described process stages, adhesive bonding to the solder mask surfaces usually takes place.

\section{Material characterization}

A cross section of the solder mask from the surface to the underlying copper was characterized by scanning electron microscope/focused ion beam (SEM/FIB) (Dual Beam FEI, Helios 600). The ion beam was generated using a gallium liquid ion metal source and operated at $30 \mathrm{kV}$. By means of energy dispersive X-ray analysis (EDS), a line scan was done along the solder mask cross sections with an acceleration voltage of $15 \mathrm{kV}$ in order to attain a good excitation of all relevant $\mathrm{x}$-ray lines, while keeping a decent lateral resolution.

Differences within the polymeric networks of the solder mask in the SM-BM and $\mathrm{SM}-\mathrm{Cu}$ configurations were investigated via infrared spectroscopy in attenuated total reflection mode (IR-ATR) utilizing Vertex 70 (Bruker) with IRscope II (germanium

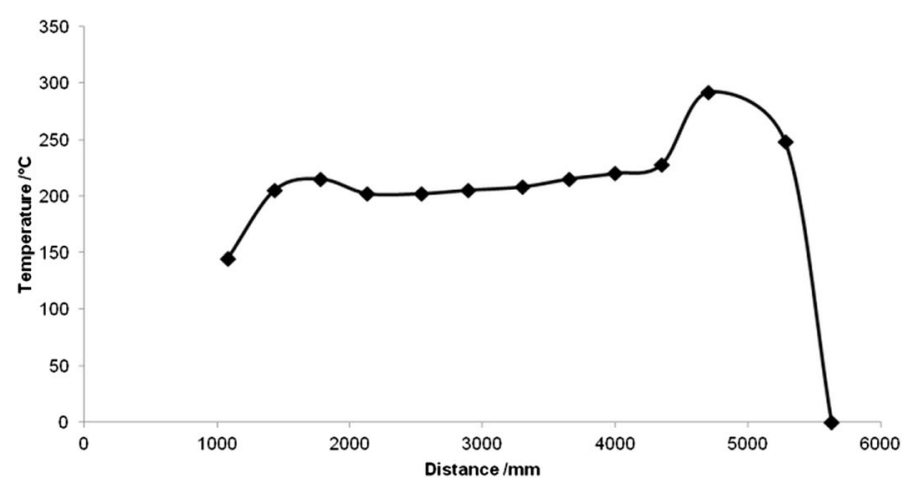

Fig. 2 Oven temperature profile: $\mathrm{PCB}$-conditioning exhibiting a measured maximum temperature of $245^{\circ} \mathrm{C}$ on the PCB surface 
crystal, 64 scans and resolution 4) within the range $700-1800 \mathrm{~cm}^{-1}$ and resulting in an information depth of $1-2 \mu \mathrm{m}$.

\section{Surface characterization}

$X$-ray photoelectron spectroscopy (XPS)

Surface composition was investigated by XPS analysis using an Escalab 220i-XL-system (Thermo VG Scientific Ltd., UK) with a monochromatic X-ray source $\left(\mathrm{Al}-K_{\alpha}\right)$. In the constant analyzer energy mode, $70 \mathrm{eV}$ pass energy for survey spectra and $20 \mathrm{eV}$ for high resolution line spectra (for example for $\mathrm{C} 1 \mathrm{~s}$ and $\mathrm{Si} 2 \mathrm{p}$ region spectra), were used. The analysis area had a diameter of approx. $0.65 \mathrm{~mm}$. Information depth can be varied from $10 \mathrm{~nm}$ (perpendicular incidence) to $5 \mathrm{~nm}$ (angle of incidence $50^{\circ}$ ). C 1s and Si 2p peakfitting were performed using synthetic Gaussian-Lorentzian components (70 and 30 \%) with a linear background subtraction. The quantitative information of the elemental surface composition was calculated based on the peak area.

\section{Time of flight secondary ion mass spectroscopy (ToF-SIMS)}

ToF-SIMS measurements were performed using a TOF-SIMS IV instrument (IONTOF GmbH, Münster, Germany) in static mode. Mass analysis was provided by a time of flight mass analyzer, energetic stimulation by a $\mathrm{Bi}_{1}{ }^{+}$ion source $(25 \mathrm{keV})$ and charge compensation by a pulsed electron flood gun. Positive and negative ion mass spectra in the mass range of $0-800 \mathrm{~m} / \mathrm{z}$ were acquired from a measurement area of $500 \mu \mathrm{m} \times 500 \mu \mathrm{m}$. For data analysis, the mass range of $0-400 \mathrm{~m} / \mathrm{z}$ was used. The surface sensitivity of the ToF-SIMS method is about $1-3$ monolayers.

To identify a species by TOF-SIMS, all physically feasible fragments of this specific component have to be characterized. Identification was accomplished by deducting potential fragments from the chemical formula and by library and mass calculating of the Ionspec software. The normalized intensity derives semi-quantitative information on the occurring species and can be determined by the ratio of the total counting rate.

Due to the amount of fragments of one component, representative fragments were selected for the data interpretation if all relevant fragments showed the same behavior.

Reproducibility of the solder mask surfaces was confirmed by distribution patterns $(5 \mathrm{~mm} \times 5 \mathrm{~mm})$ with ToF-SIMS. For this purpose, three samples were measured two times and exhibited a homogenous component distribution of the identified species in all cases. Due to the homogeneity of the sample surface and the quality of the peak fits, proven by standard deviation calculation of six different spectra, the number of ToFSIMS measurements was limited to two repetitions, each with positive and negative polarization. Correlation of the ToF-SIMS measurements with the respective surface energy measurements, including standard deviations, and the XPS results confirmed that no deviations within one sample type occurred.

\section{Optical contact angle measurement (OCA)}

Optical contact angle measurements were performed using an EasyDrop standard (Krüss GmbH, Hamburg, Germany) with Drop Shape Analysis DSA1 software equipment. Three fluids were used with the sessile drop method: water (HPLC grade) from Sigma-Aldrich (CAS: 7732-18-5), diiodomethane with $99 \%$ purity from Sigma-Aldrich 
(CAS: 75-11-6) and ethylene glycol p.a. with $>99.5 \%$ (GC) from Fluka Analytics (CAS: 107-21-1). By using the Owens-Wendt-Kaelble-Rabel (OWKR)-method [17], both the polar and disperse parts of the surface energy were determined. For this purpose, nine single drops of each fluid were applied on the sample surface and the optical contact angle was measured. The surface sensitivity of OCA is one monolayer.

\section{Adhesion testing}

For adhesion testing, the pull-off strength was determined with respect to ISO 4624 in a tensile testing machine (T1-FR010TN.A50, Zwick GmbH \& Co, Ulm, Germany). For this purpose, an aluminum stud with a $70.5 \mathrm{~mm}^{2}$ contact area was attached to the solder mask surface by using a heat curing epoxy adhesive (SMT-Adhesive PD955M, Heraeus Materials Technology GmbH \& Co.KG, Hanau, Germany). The curing condition was $120^{\circ} \mathrm{C}$ for $6 \mathrm{~min}$. Ten test samples were prepared and tested for each solder mask in each configuration (SM-BM and SM-Cu) and the failure mode was documented.

\section{Results and discussion}

\section{SEM/FIB-EDS analysis of the solder mask bulk volume}

The presence of copper in the solder mask type A matrix was analyzed using SEM/FIBEDS. A cross section of the solder mask was generated through FIB analysis. The EDS line scans exhibited the presence of copper within the polymer network with decreasing content from the copper/solder mask interface up to the solder mask surface (Fig. 3). The copper originates from the copper base layer underneath, which was chemically roughened in a copper micro-etching step, for example, through a mixture of sulfuric acid and hydrogen peroxide before solder mask application. Table 1 summarizes the copper content of four different positions along the line scan marked by index 1-4. As displayed in Fig. 2 (right), the copper content decreases rapidly within the solder mask to approximately $22 \mathrm{wt} \%$ and decreases slightly towards the solder mask surface. Hence, even at the solder mask near surface region, the $\mathrm{Cu}$ content still is about $10 \mathrm{wt} \%$ according to the extracted spectra. In the case of the solder mask applied on base material, no copper was measured in a cross section by EDS.

It has to be noted that in solder mask formulations, a copper complex of phthalocyanine (chemical formula: $\mathrm{CuC}_{32} \mathrm{Cl}_{16-\mathrm{n}} \mathrm{H}_{\mathrm{n}} \mathrm{N}_{8}$; CAS-No: 1328-53-6) is present as pigment.
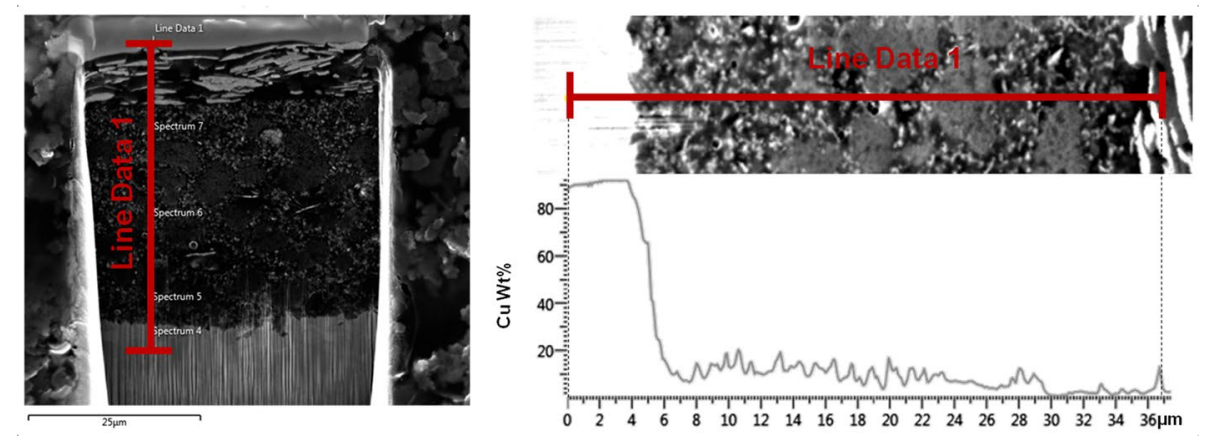

Fig. 3 SEM/FIB-EDS: position of the line scan along the solder mask cross section with the positions of the four extracted spectra (left) and the locally resolved copper content in weight percent of the line scan (right) 
Table 1 Copper concentration of the individual spectra through the solder mask profile displayed in Fig. 3

\begin{tabular}{lllll}
\hline Label & Spectrum 1 & Spectrum 2 & Spectrum 3 & Spectrum 4 \\
\hline Cu/wt\% & 80 & 22 & 12 & 9 \\
\hline
\end{tabular}

Base level of copper concentration arises from the pigment phthalocyanine-copper complex and is calculated to approx. $0.1-0.3 w t \%$

This copper complex leads to a ground level of copper concentration in the solder mask matrix. Deriving from the pigment content, $2-5 \mathrm{wt} \%$ of the copper complex corresponds to $0.1-0.3 \mathrm{wt} \%$ copper in the solder mask formulation and leads to an expected value of $0.03-0.09$ at\%. Additionally, in the cross section, just a part of the total solder mask volume is measured. Therefore, the concentration of copper as pigment is under the detection limit of EDS, which for the $K_{\alpha}$-line at $15 \mathrm{kV}$ accelerating voltage is around 0.3$0.4 \mathrm{wt} \%$. Since it is known by the investigations of Parry et al. [18] that phthalocyanine compounds are used to cure epoxy-novolac, the presence of reactive amines, carboxyl acids or carboxylic ester groups on the phenol rings of the complex influence the ring opening reaction of the epoxy group. Additionally, Achar et al. [19] postulated the need for solubility of the copper phthalocynanine complex in aprotic solvents and the resulting introduction into the epoxy resin. Since the copper phthalocyanine complex exhibits chlorine at the phenol rings instead of reactive groups as mentioned by Parry et al. [18], no interaction between the pigment and the epoxy curing is expected.

Considering various investigations in the past $[5,6,10-12,20]$, the presence of copper may lead to the formation of copper complex species with large expansion in the polymer bulk [8], resulting in a modified curing and cross-linking behavior of the polymeric network. The impact of the measured copper content on the surface chemistry as well as on the adhesion behavior to an epoxy adhesive, will be evaluated in the following section.

\section{IR-ATR measurements for cross linking investigation}

Differences in the cross linking behavior are supposed to be visible by comparing oneto-one the IR-ATR spectra of the solder masks in SM-BM and SM-Cu configuration, since an accelerated or decelerated cross-linking should provide different contents of epoxy and/or acrylate terminated groups in the bulk material. Representatively, Fig. 4 displays the overall spectra of solder mask type A in the range of $700-4000 \mathrm{~cm}^{-1}$ in both the SM-BM and SM-Cu configuration. Detailed analysis revealed no significant differences within the spectra. Alkyl stretching at $2800-3000 \mathrm{~cm}^{-1}$ exhibited small deviation, which was attributed to the presence of copper caused by the copper gradient, by comparing to spectra of the bare copper. Total correlation between the spectra of the two configurations SM-BM and SM-Cu was determined to $99.93 \%$. Carbonyl stretching in the range of $1600-1700 \mathrm{~cm}^{-1}$ and the fingerprint area exhibits analogous shapes and intensity. Thus, the presence of significant amounts of copper in the coating seems to have minor or no influence on the network formation and the degree of cross-linking. 


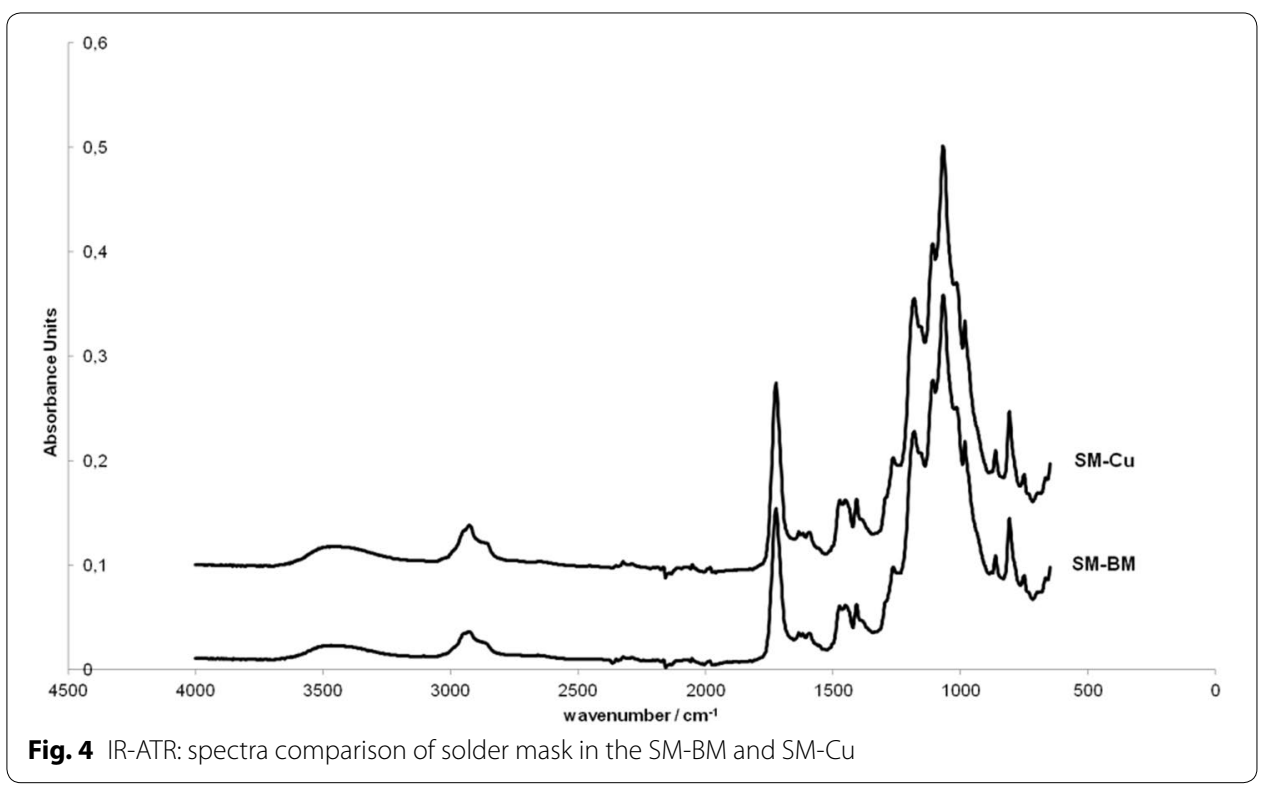

\section{ToF-SIMS and XPS measurements for surface characterization}

Representatively, Fig. 5 displays typical fragments of the ToF-SIMS spectra of solder mask type A in both the SM-BM and SM-Cu configurations. All identified polar and non-polar fragments (Fig. 5a) on these surfaces showed no significant deviations. Additionally, the PDMS fragments (Fig. 5b), sourcing from typical additives in the solder mask formulation like anti-foaming and wetting agents, showed the same signal intensity. This also supports the assumption that there are no differences in the cross-linking behavior, since an increased cross-linking density as well as the presence of polar groups due to the interaction with PDMS, should lead to lower PDMS contents at the surface due to inhibited PDMS migration.

Only the normalized intensities of copper isotopes ${ }^{63} \mathrm{Cu}^{+}$and ${ }^{65} \mathrm{Cu}^{+}$exhibited significant differences between the surfaces of the two configurations, with a higher normalized intensity for the SM-Cu configuration. The existence of copper on the surface of SM-BM specimens was attributed to both the presence of copper containing pigments and the OSP-copper complex layer [21] (see Fig. 1), sourcing from the passivation step within the PCB manufacturing process, which generates a basic copper level in both configurations on the surface. The higher level in the case of SM-Cu arises from the additional $\mathrm{Cu}$ layer underneath. As a consequence, the residues caused by OSP in Fig. 5a like $\mathrm{C}_{3} \mathrm{H}_{2} \mathrm{Cu}^{+}, \mathrm{C}_{6} \mathrm{H}_{5} \mathrm{~N}_{2} \mathrm{Cu}^{+}$and $\mathrm{C}_{10} \mathrm{H}_{7} \mathrm{SN}_{3} \mathrm{Cu}^{+}$showed the same signal intensities in both configurations. Since the copper of the OSP-Cu complex could only be detected with the ToF-SIMS method, the copper content is in the range of 100-1000 ppm. Supporting the ToF-SIMS observations relating to the solder mask surface chemistry, the concentrations of the elements carbon, oxygen, nitrogen and silicon on the solder mask surface, measured with XPS on the solder mask surface for quantitative information, showed no significant variance as presented in Table 2. Figure 6 summarizes the various copper sources and the detection methods depending on the information depth and location. 
(a)

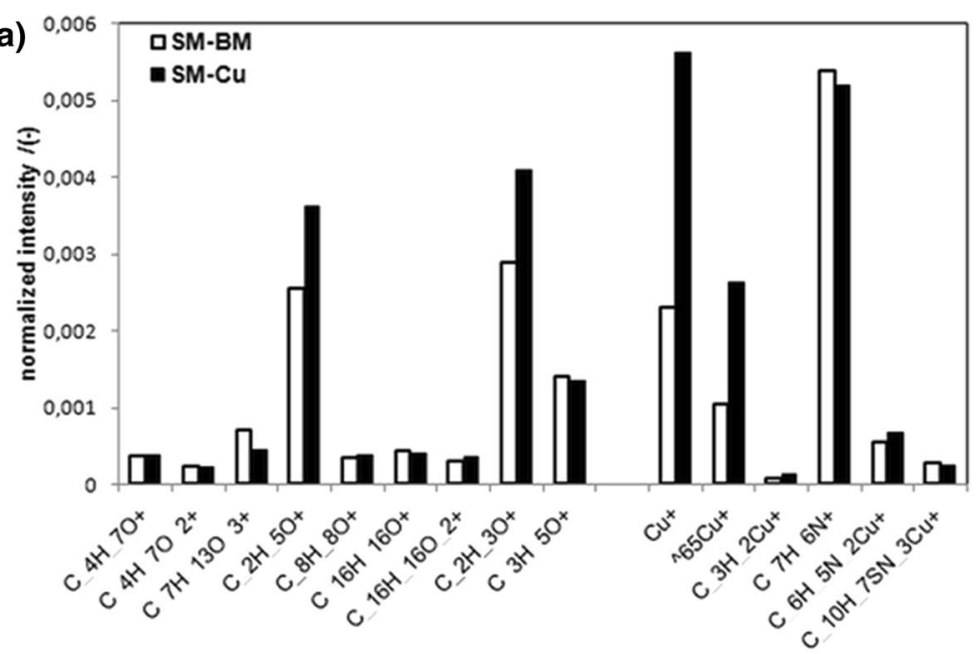

(b)

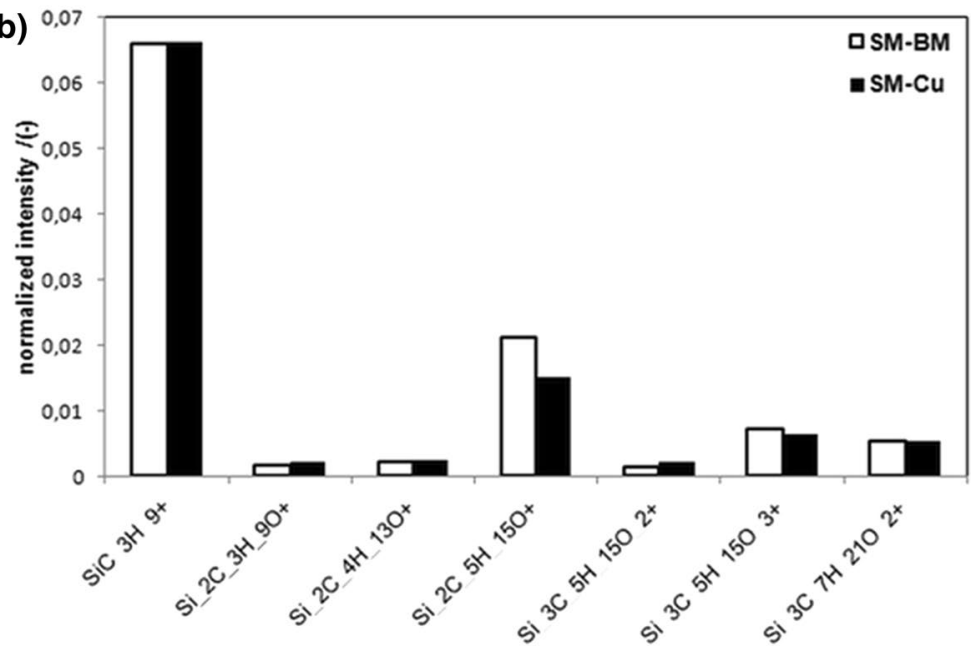

Fig. 5 ToF-SIMS: normalized intensities of the polar and non-polar groups (a) and the PDMS fragments (b) at the surface of solder mask A, also representative for solder mask type B

Table 2 XPS measurements on the solder mask surfaces of different solder mask types in the SM-BM and SM-Cu configurations

\begin{tabular}{llllll}
\hline Solder mask type & Configuration & C/at\% & O/at\% & Si/at\% & N/at\% \\
\hline A & SM-BM & 64.8 & 30.0 & 3.1 & 1.8 \\
& SM-C & 66.8 & 28.0 & 2.9 & 1.6 \\
B & SM-BM & 65.8 & 29.0 & 3.4 & 1.3 \\
& SM-C & 67.4 & 27.0 & 3.2 & 1.6 \\
\hline
\end{tabular}

Atomic percentages are displayed for the elements carbon, oxygen, silicon and nitrogen

In Fig. 7, the total surface energies of the various solder mask types for the different configurations SM-BM and SM-Cu are displayed. Since the surface energy of the substrate is a necessary condition regarding adhesive wetting, the deviation between the configurations is negligible. Still, there are other influences which also determine the adhesion between an adhesive and the respective substrate. These are, for example, 

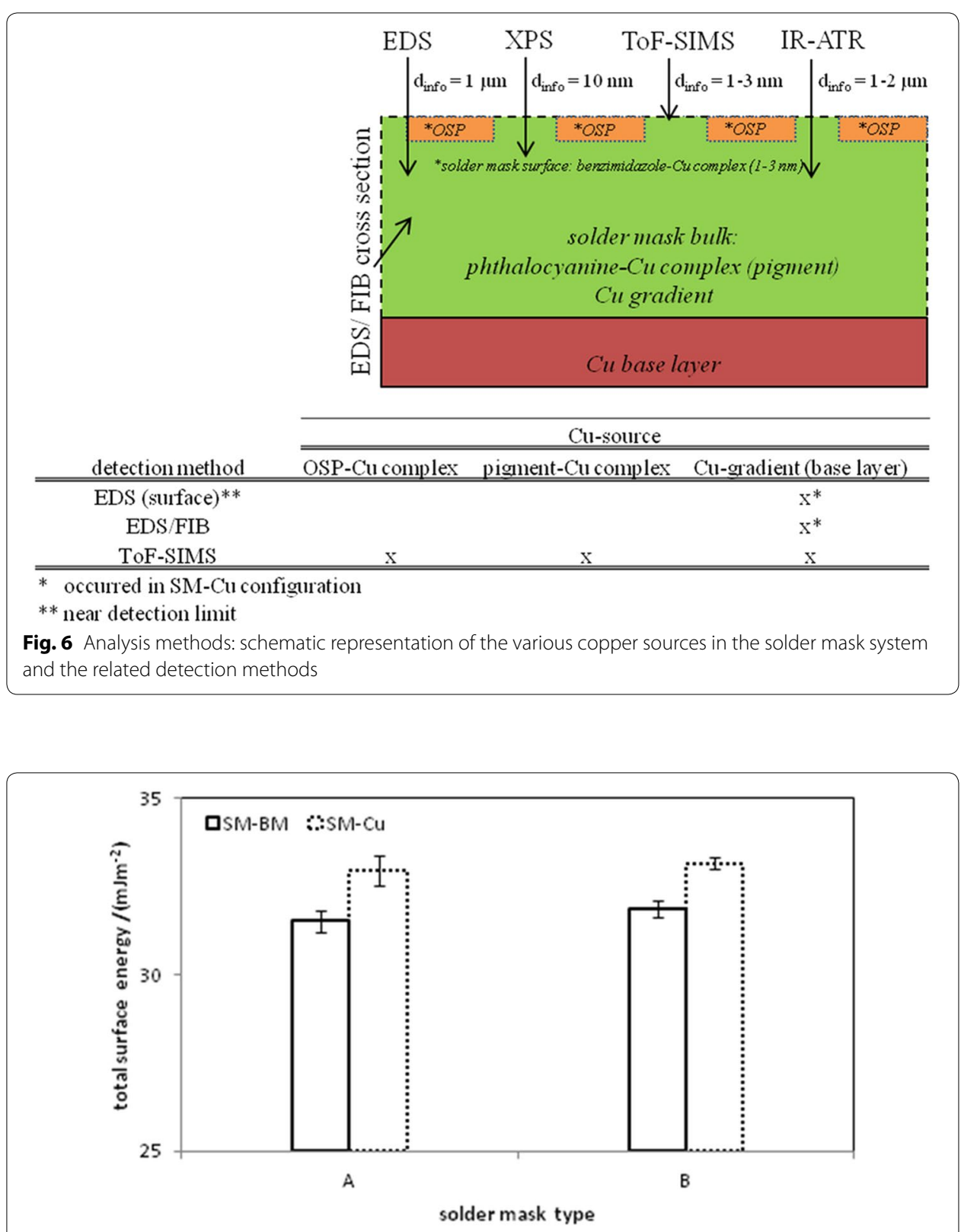

Fig. 7 Surface energy from OCA: comparison of the solder mask types A and B within the SM-BM and SM-Cu configurations

surface roughness of the substrate [22] and formation of a weak boundary layer [23] within the substrate to the adhesive interface. In this study, the base material and the copper substrate were kept constant and treated similarly prior to solder mask application. Consequently, resulting differences in any observation may arise from the solder mask type and are not caused by process influences.

\section{Adhesion testing}

Figure 8 shows the adhesion strength of the two different solder mask types in the two configurations, given as pull-off tension. The copper layer underneath has no significant 


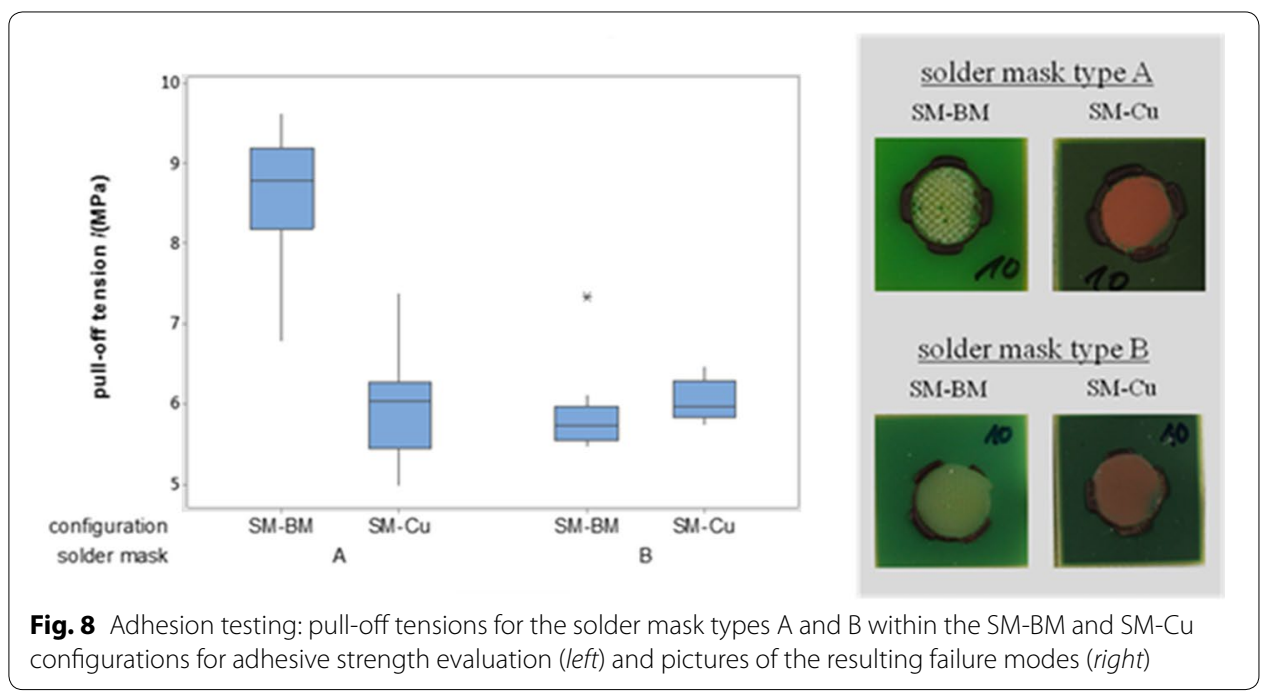

influence on the adhesion between the solder mask and the epoxy adhesive. All observed failures of the bonded samples occurred within (between the glass fibers and the epoxy resin of the base material) or to the base material (configuration SM-BM) or at the interface of solder mask to copper (configuration SM-Cu). A Two-Sample $T$ test with a confidence interval of $95 \%$ was carried out for statistical considerations. With a confidence of $95 \%$, the difference between solder mask A in SM-BM configuration and in SM-Cu configuration is $2-3 \mathrm{MPa}$ higher in the SM-BM configuration than in the SM-Cu configuration ( $\mathrm{p}$ value $<0.001$ ). In this case, the base material exhibited a cohesive-like failure of the interface epoxy-resin/glass fiber of the FR4-laminate.

Regarding the SM-BM configuration with a confidence of $95 \%$, the difference between the solder mask A and solder mask B within the SM-BM configuration is $2-3 \mathrm{MPa}$ higher in solder mask A than in solder mask B (p value $<0.001$ ). Since the substrates were treated in the same way by micro-etching before solder mask application, differences in the substrate roughness could be excluded with regards to adhesion differences between solder mask A and B. Thus, the differences must be associated with the individual solder mask formulation.

The higher pull-off strength of solder mask A compared to that of solder mask B in the SM-BM configuration as well as both solder masks in the SM-Cu configuration, may be attributed to the failure mode. Akisanya et al. [24] reported a higher bonded joint toughness if the failure mode exhibits a serrated crack path compared to a flat crack path at the interface of two components. In terms of the crack path within the base material, the crack has to follow the structure of the glass fiber mat resulting in a longer crack path.

Scattering of tension values can be explained by scattering in the failure mode, while no adhesive failure of the solder mask/epoxy adhesive interface occurred in the SM-BM or in the SM-Cu configuration. The statistical variance in the solder mask type B configuration SM-BM was related to the particular cohesive failure of the solder mask itself. No adhesive failure to the epoxy adhesive was observed, but there was residue of solder mask on the interface base material/solder mask. 
The chemistry of the resin components of the two solder mask types are comparable (epoxy-acrylate), still, the thermal curing agents are different and exhibit the option of copper complex formation between the amine or the anhydride and the copper of the copper base layer, leading to accelerated or decelerated curing kinetics and differences in the cross-linking density. However, as displayed in Fig. 8, the presence of copper leads neither to a cohesive failure within the solder mask bulk, nor to differences in the strength values within the SM-Cu configurations of the two solder mask types. Therefore, no impact of the presence of copper within the solder mask bulk to the network formation or the adhesion behavior might be stated.

\title{
Conclusion
}

Since the modifying effect on epoxy curing due to metal and coordination components and its effect on network structure are already documented in literature [5, 7, 10-12, 20], the impact of the presence of copper in a cured solder mask on the adhesion behavior was investigated in this study. The presence of copper in the epoxy acrylate bulk volume exhibited no changes in the network formation in the surface near region (within $2 \mu \mathrm{m}$ information depth). Differences in the surface chemistry concerning the copper content from the top most monolayer to $10 \mathrm{~nm}$ in the surface near region do not affect the wetting and adhesion behavior to an epoxy adhesive. Breakage of the solder masks from the substrate base material and copper base layer exhibits the same pull-off strength values if the interface glass fiber/epoxy resin within the base material is not affected. The failure mode in this case includes a serrated crack path along the glass fiber mat leading to higher pull-off strength values. The mechanical strength of the solder mask/copper base layer interface is not influenced by the different copper complex formations of amine and anhydride based curing agents.

\begin{abstract}
Authors' contributions
$\mathrm{CH}$ coordinated the surface analysis, carried out sample preparation and, the time of flight-secondary ion mass spectroscopy (ToF-SIMS), optical contact angle (OCA) measurements, adhesion testing and conducted the interpretation of data, as well as the drafting and revising of the manuscript. SM participated in drafting the experimental setup, data interpretation of the adhesion testing and revising the manuscript. TF supported the ToF-SIMS and X-ray photoelectron spectroscopy (XPS) measurements and data interpretation and aided in the revising of the manuscript. KT carried out the focused ion beam (FIB) cross sections and the energy dispersive spectroscopy (EDS) measurements and data interpretation. BM participated in the conception of the investigations, drafting and revising the manuscript and providing information about adhesive bonding processes. All authors read and approved the final manuscript.
\end{abstract}

\footnotetext{
Author details

${ }^{1}$ Robert Bosch GmbH, Postfach 3002 40, 70442 Stuttgart, Germany. ${ }^{2}$ Fraunhofer Institute for Manufacturing Technology and Advanced Materials IFAM, Wiener Str. 12, 28359 Bremen, Germany.

Competing interests

The authors declare that they have no competing interests.
}

Received: 25 August 2015 Accepted: 8 October 2015

Published online: 13 October 2015

\footnotetext{
References

1. IPC-HDBK-840. IPC Association Connecting Electronics Industries; 2006.

2. Park YJ, Lim DH, Kim HJ, Park DS, Sung IK. UV- and thermal-curing behaviors of dual-curable adhesives based on epoxy acrylate oligomers. Int J Adhes Adhes. 2009;29(7):710-7. doi:10.1016/j.ijadhadh.2009.02.001.

3. Park WH, Lee JK, Kwon KJ. Cure behavior of an epoxy-anhydride-imidazole system. Polym J. 1996;28(5):407-11. doi:10.1295/polymj.28.407

4. Pham HQ, Marks MJ. Epoxy resins in Ullman's encyclopedia of industrial chemistry (online version). Weinheim: WileyInterscience; 2005.
} 
5. Possart W, Krüger JK, Wehlack C, Müller U, Petersen C, Bactavatchalou R, Meiser A. Formation and structure of epoxy network interphases at the contact to native metal surfaces. C R Chim. 2006;9(1):60-79. doi:10.1016/j. crci.2005.04.009.

6. Marsh J, Minel L, Barthés-Labrousse MG, Gorse D. Interaction of epoxy model molecules with aluminium, anodised titanium and copper surfaces: an XPS study. Appl Surf Sci. 1998;133(4):270-86. doi:10.1016/S0169-4332(97)00789-7.

7. Hamerton I, Howlin BJ, Jones JR, Liu S, Barton JM. Effect of complexation with copper (II) on cured neat resin properties of a commercial epoxy resin using modified imidazole curing agents. J Mater Chem. 1996;6(3):305-10. doi:10.1039/JM9960600305.

8. Roche AA, Bouchet J, Bentadjine S. Formation of epoxy-diamine/metal interphases. Int J Adhes Adhes. 2002;22(6):431-41. doi:10.1016/S0143-7496(02)00021-0.

9. Bentadjine S, Petiaud R, Roche AA, Massardier V. Organo-metallic complex characterization formed when liquid epoxy-diamine mixtures are applied onto metallic substrates. Polymer. 2001;42(14):6271-82. doi:10.1016/ S0032-3861(01)00034-9.

10. Dowbenko R, Anderson CC. US Patent US3678007. 1972

11. Dowbenko R, Anderson CC, Chang WH. US Patent US3677978. 1972.

12. Dowbenko R, Anderson CC, Chang WH. UK Patent GB1204834. 1970.

13. Awaja F, Gilbert M, Kelly G, Fox B, Pigram PJ. Adhesion of polymers. Prog Polym Sci. 2009;34(9):948-68. doi:10.1016/j. progpolymsci.2009.04.007.

14. Bosshart WC. Printed circuit boards: design and technology. Noida: Tata McGraw Hill; 1983.

15. Tong KH, Ku MT, Hsu KL, Tang Q, Chan CY, Yee KW. The evolution of organic solderability preservative (OSP) process in PCB application. In: Microsystems, Packaging, Assembly and Circuits Technology Conference (IMPACT), 2013 8th International. 2013.

16. Yoshida Shuji, Ishida Hatsuo. A study on the orientation of imidazoles on copper as corrosion inhibitor and possible adhesion promoter for electric devices. J Chem Phys. 1983:78(11):6960-9. doi:10.1063/1.444644.

17. Manual of contact angle measurement systems. Kruess GmbH. p. 131.

18. Parry HL. US Patent 3301814. 1967.

19. Achar BN, Fohlen GM, Parker JA. Metal(II) 4,4',4", $4^{\prime \prime \prime}$-phthalocyanine tetramines as curing agents for heat resistant epoxy formulations. J Polym Sci Polym Chem Ed. 1983;21(5):1505-16. doi:10.1002/pol.1983.170210522.

20. Hamerton I, Howlin BJ, Jepson P. Metals and coordination compounds as modifiers for epoxy resins. Coord Chem Rev. 2002;224(1-2):67-85. doi:10.1016/S0010-8545(01)00393-9.

21. Annenkov W, Danilovtseva EN, Saraev W, Mikhaleva Al. Complexation of copper(II) ions with imidazole-carboxylic polymeric systems. J Polym Sci Part A Polym Chem. 2003;41(14):2256-63. doi:10.1002/pola.10769.

22. Packham DE. Surface energy, surface topography and adhesion. Int J Adhes Adhes. 2003;23(6):437-48. doi:10.1016/ S0143-7496(03)00068-X.

23. Bikerman Jacob J. Causes of poor adhesion: weak boundary layers. Ind Eng Chem. 1967;59(9):40-4. doi:10.1021/ ie51403a010.

24. Akisanya AR, Fleck NA. Brittle fracture of adhesive joints. Int J Fract. 1992;58(2):93-114. doi:10.1007/BF00019971.

\section{Submit your manuscript to a SpringerOpen ${ }^{\circ}$ journal and benefit from:}

- Convenient online submission

- Rigorous peer review

- Immediate publication on acceptance

- Open access: articles freely available online

- High visibility within the field

- Retaining the copyright to your article

Submit your next manuscript at $>$ springeropen.com 